\title{
The spectrum of the very massive binary system WR 20a (WN6ha + WN6ha): Fundamental parameters and wind interactions ${ }^{\star}$
}

\author{
G. Rauw ${ }^{1, \star \star}$, P. A. Crowther ${ }^{2}$, M. De Becker ${ }^{1}$, E. Gosset ${ }^{1, \star \star}$, Y. Nazé ${ }^{1}$, \\ H. Sana ${ }^{1, \star \star \star}$, K. A. van der Hucht ${ }^{3,4}$, J.-M. Vreux ${ }^{1}$, and P. M. Williams ${ }^{5}$ \\ ${ }^{1}$ Institut d'Astrophysique et de Géophysique, Université de Liège, Allée du 6 Août 17, Bât. B5c, 4000 Liège, Belgium \\ e-mail: rauw@astro.ulg.ac.be \\ 2 Department of Physics \& Astronomy, University of Sheffield, Hicks Building, Hounsfield Rd., Sheffield, S3 7RH, UK \\ 3 SRON National Institute for Space Research, Sorbonnelaan 2, 3584 CA Utrecht, The Netherlands \\ 4 Astronomical Institute Anton Pannekoek, University of Amsterdam, Kruislaan 403, 1098 SJ Amsterdam, The Netherlands \\ 5 Institute for Astronomy, University of Edinburgh, Royal Observatory, Blackford Hill, Edinburgh, EH9 3HJ, UK
}

Received 7 October 2004 / Accepted 23 November 2004

\begin{abstract}
We analyse the optical spectrum of the very massive binary system WR 20a (WN6ha + WN6ha). The most prominent emission lines, $\mathrm{H} \alpha$ and $\mathrm{He}$ II $\lambda$ 4686, display strong phase-locked profile variability. From the variations of their equivalent widths and from a tomographic analysis, we find that part of the line emission probably arises in a wind interaction region between the stars. Our analysis of the optical spectrum of WR 20a indicates a reddening of $A_{\mathrm{V}} \simeq 6.0$ mag and a distance of $\sim 7.9 \mathrm{kpc}$, suggesting that the star actually belongs to the open cluster Westerlund 2 . The location of the system at $\sim 1.1 \mathrm{pc}$ from the cluster core could indicate that WR 20a was gently ejected from the core via dynamical interactions. Using a non-LTE model atmosphere code, we derive the fundamental parameters of each component: $T_{\text {eff }}=43000 \pm 2000 \mathrm{~K}, \log L_{\mathrm{bol}} / L_{\odot} \simeq$ $6.0, \dot{M}=8.5 \times 10^{-6} M_{\odot} \mathrm{yr}^{-1}$ (assuming a clumped wind with a volume filling factor $f=0.1$ ). Nitrogen is enhanced in the atmospheres of the components of WR 20a, while carbon is definitely depleted. Finally, the position of the binary components in the Hertzsprung-Russell diagram suggests that they are core hydrogen burning stars in a pre-LBV stage and their current atmospheric chemical composition probably results from rotational mixing that might be enhanced in a close binary compared to a single star of same age.
\end{abstract}

Key words. stars: binaries: spectroscopic - stars: early-type - stars: fundamental parameters - stars: individual: WR 20a stars: Wolf-Rayet

\section{Introduction}

In a recent letter, our team reported the discovery of the binary nature of WR 20a (Rauw et al. 2004, hereafter Paper I). We found that the system consists most probably of two WN6ha stars with almost identical spectra revolving around each other in $~ 3.7$ days. Our orbital solution indicated very large minimum masses of about $70 M_{\odot}$ for each star and we predicted that the binary should display photometric eclipses (Paper I). These results triggered a prompt photometric study of WR 20a by Bonanos et al. (2004) who actually detected the eclipses in their $I$-band photometry hence confirming, and slightly improving, the orbital period of the system. Combining the value of the orbital inclination of $74.5^{\circ} \pm 2^{\circ}$ derived by Bonanos et al. with the radial velocity data from Paper I yields masses

* Based on observations collected at the European Southern Observatory, La Silla, Chile.

$\star \star$ Research Associate FNRS, Belgium.

$\star \star \star$ Research Fellow FNRS, Belgium. of about $80 M_{\odot}$ for both stars, thus making WR 20a the most massive binary with well determined masses so far.

In this paper, we present an in-depth study of the spectrum of WR 20a in order to constrain the properties of its WolfRayet (WR) components. Information on the mass-loss rates and the chemical composition of the stellar winds as well as on the stellar temperatures and luminosities are crucial for understanding the evolutionary stage of such an exceptional system. In addition, given the extreme properties of the stars in WR 20a and their small separation, various interactions such as Roche lobe overflow or a wind-wind collision may occur in this system. These interactions imply the motion of high-density material between the stars that can best be traced through a study of the phase-locked variations of the emission lines that are formed through recombination in the high-density material.

This paper is organised as follows. Our observations are presented in Sect. 2, whilst we discuss the features of the green and red spectrum of WR 20a in Sect. 3. Section 4 deals with the analysis of the line profile variability that affects the $\mathrm{H} \alpha$ 


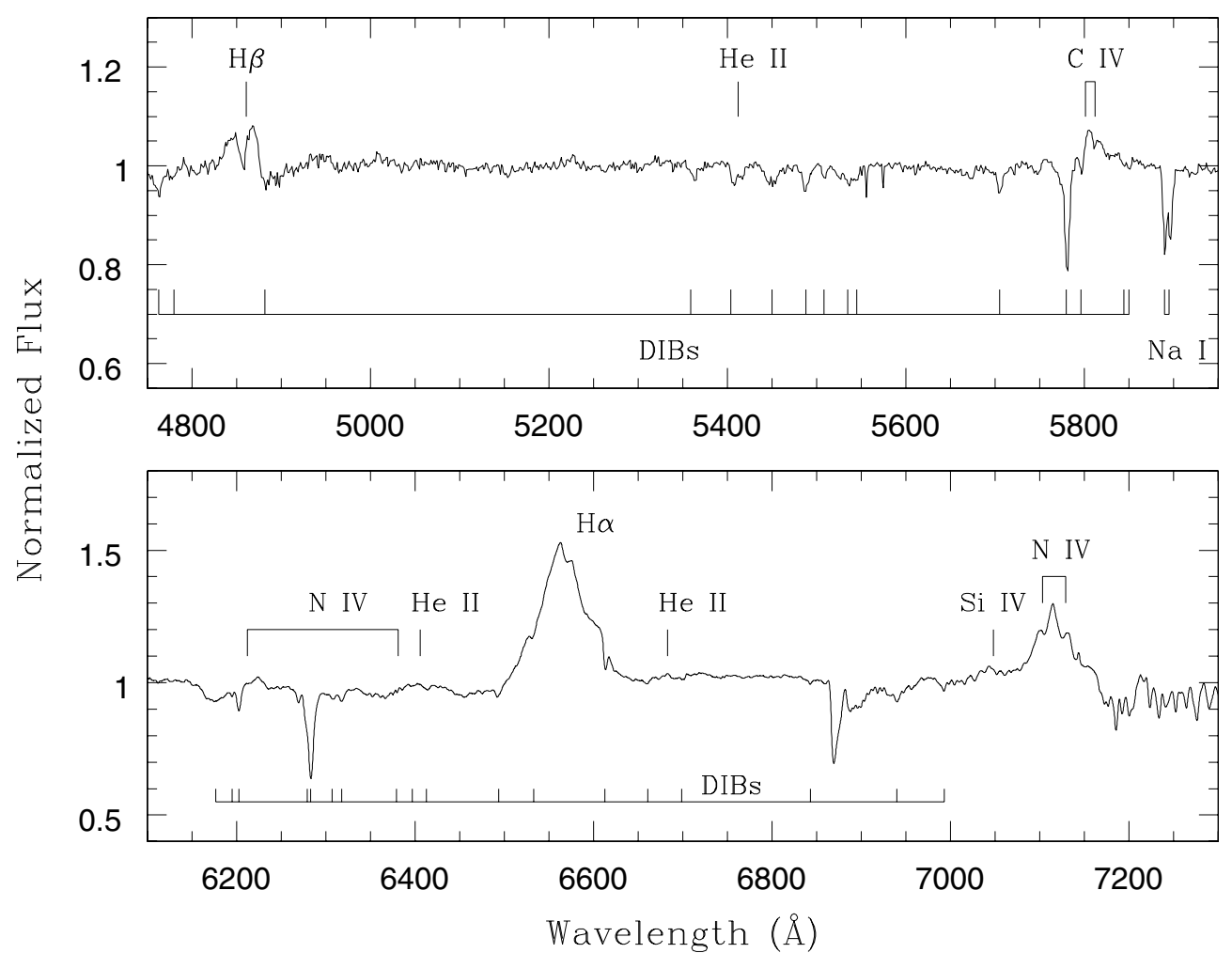

Fig. 1. Green and red spectrum of WR 20a. The top panel corresponds to the spectrum observed at phase 0.895 (HJD 2453 179.472), whilst the lower panel corresponds to phase 0.798 (HJD 2453 042.734). In addition to the telluric absorptions, the bulk of the absorption features in these spectral domains are due to diffuse interstellar bands (the identifications are taken from Herbig 1995). The stellar spectrum is dominated by strong emission lines of $\mathrm{H} \alpha$ and several blends due to N IV lines. Note also the absence of He I $\lambda 5879$ and the presence of CIV $\lambda \lambda 5801$, 5812 in emission.

and He II $\lambda 4686$ emissions. In Sect. 5, we use a state of the art model atmosphere code to derive the fundamental properties of the stars and their winds. Finally, in Sect. 6, we discuss the implications of our results and we highlight some future perspectives to solve some of the still open questions related to WR 20a.

\section{Observations}

In addition to the blue and violet spectra presented in Paper I, we analyse here a set of red spectra of WR 20a. Ten medium resolution spectra were obtained in January-February 2004 with the EMMI instrument mounted on ESO's $3.5 \mathrm{~m}$ New Technology Telescope (NTT) at La Silla. The EMMI instrument was used in the RILD low dispersion spectroscopic mode with grism \# 6 (600 grooves $\mathrm{mm}^{-1}$ ) providing a wavelength coverage from about 5750 to $8670 \AA$. The slit width was set to 1 arcsec and the exposure times were $10-15$ min yielding a $S / N$ ratio of about 200 . The spectral resolution as determined from the $F W H M$ of the lines of the He-Ar comparison spectra was about $4.0 \AA$. The data were reduced in the standard way using the long context of the MIDAS package.

Another low resolution spectrum was obtained on June 22, 2004 with the same equipment except for the grism, where we used grism \#5 instead (600 grooves $\mathrm{mm}^{-1}$ ), which provides a wavelength coverage from 3800 to $7020 \AA$ and a spectral resolution of $5.0 \AA$ (FWHM of the He-Ar lines). The weather conditions during that night were very good and we also obtained an observation of the spectro-photometric standard star LTT 9239 (Stone \& Baldwin 1983; Hamuy et al. 1992) that we used to perform a relative flux calibration of the spectrum of WR 20a.

\section{The optical spectrum of WR 20a}

The blue-violet spectrum of WR 20a is illustrated in Paper I and the reader is referred to that paper for a detailed description. In this section we shall rather focus on the spectral features at wavelengths from 4800 to $7200 \AA$ that provide important clues on the nature of the stars. This part of the spectrum is illustrated in Fig. 1.

First of all, we note the important number of relatively strong diffuse interstellar bands (DIBs) that account actually for most of the absorption features in the green - red spectrum of WR 20a. Although the strength of most DIBs and the $E(B-V)$ colour excess are not systematically correlated, except for a few features (Herbig 1995), the strengths of the DIBs in the spectrum of WR 20a is a clear indication of a rather heavy interstellar absorption. Indeed, we can get a lower limit to the colour excess of WR 20a by comparing the strength of those DIBs that correlate best with $E(B-V)$ (at 5780 and $5797 \AA$ ) with their strength in the well studied interstellar spectrum of HD 183143 (B7 I, $E(B-V)=1.28$, see Herbig 1995): since the DIBs are stronger in the spectrum of WR 20a, we conclude that $E(B-V) \geq 1.3$ at least. 

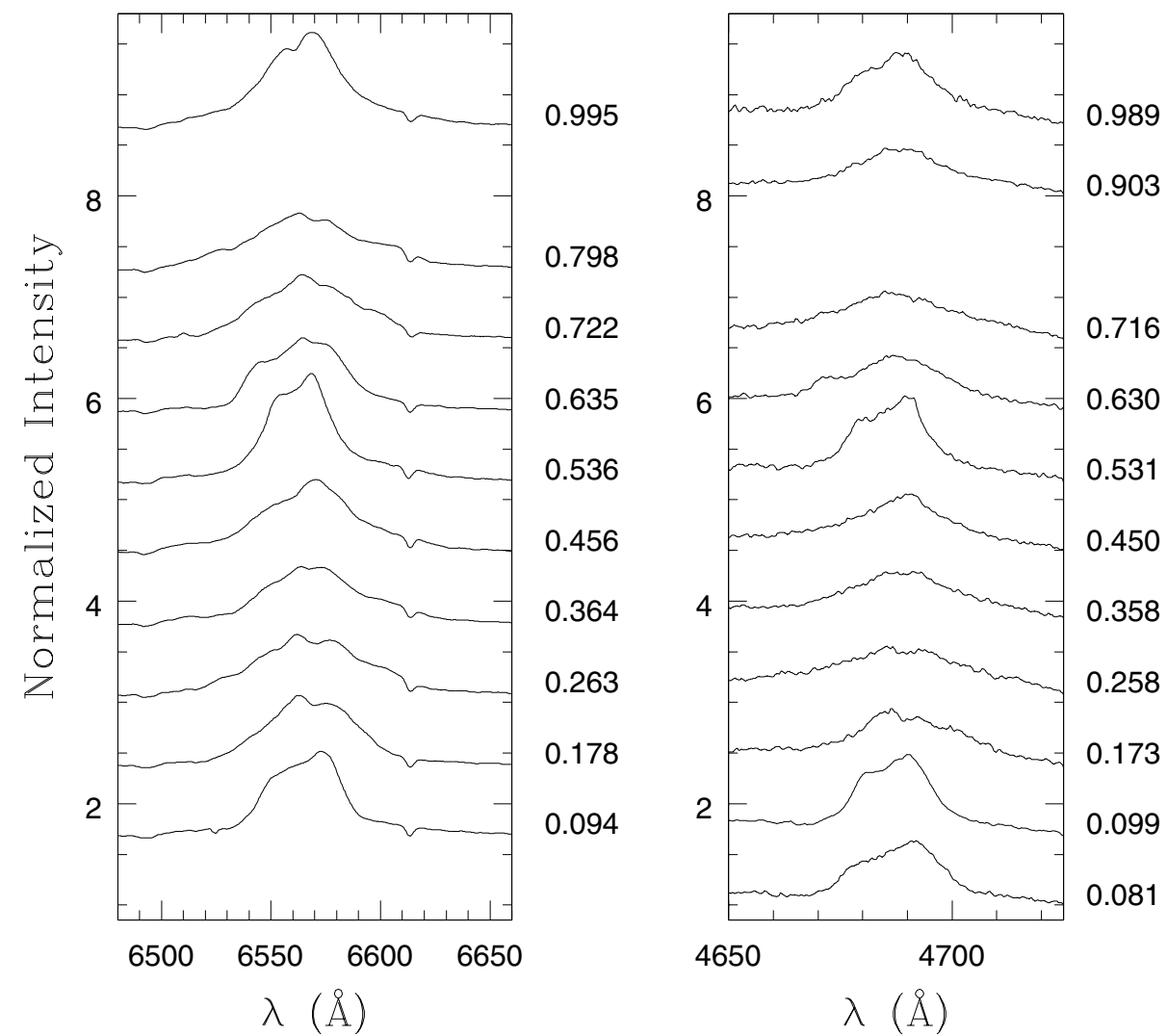

Fig. 2. Line profile variability of the $\mathrm{H} \alpha$ (left) and He II $\lambda 4686$ (right) emission lines in the spectrum of WR 20a. The normalized spectra are vertically shifted by 0.7 . The orbital phases of the observations, computed using the ephemeris of Bonanos et al. (2004), are given on the right of each panel. Note the striking similarity between the profiles of the two lines at similar orbital phases.

The most prominent stellar features are emission lines of $\mathrm{H} \alpha, \mathrm{H} \beta, \mathrm{C}$ IV $\lambda \lambda$ 5801, 5812 and N IV $\lambda \lambda$ 6212-20, 6381, $7103-29$. The $\mathrm{H} \beta$ line was observed at orbital phase 0.895 , i.e. shortly before conjunction, and the double-peaked morphology of the line can therefore not be attributed to the deblending of the lines of the two components. Without a knowledge of the variations of this line, it is difficult to provide a unique interpretation of the double-peaked profile. It could either originate in a wind interaction zone, as is likely to be the case of the two peaks seen in $\mathrm{H} \alpha$ and He II $\lambda 4686$ (see Sect. 4), or alternatively it could be intrinsic to the stars. The latter interpretation would be supported by the shape of the line in the spectrum of WR 25, another WN6ha star (see e.g. Crowther et al. 1995) as well as by the synthetic profile simulated with a model atmosphere code (see Sect. 5 and Fig. 6). The presence of $\mathrm{H} \beta$ in emission further favours a WN6ha rather than an O3 If*/WN classification for both components of WR 20a.

The CIV $\lambda \lambda$ 5801, 5812 blend has an equivalent width of about $1.0 \pm 0.1 \AA$ on our spectrum ${ }^{1}$. The presence of this line in the spectrum of WR 20a along with the weakness of He I $\lambda 5876$ was first noted by Shara et al. (1999) who classified WR 20a accordingly as a WN7:h/C star. Finally, we note that the He II $\lambda 5412$ line is seen in absorption. This is not

${ }^{1}$ We note that this value is likely to be a lower limit on the actual line strength because of the contamination of the blend by several DIBs. surprising since the blue spectra presented in Paper I already indicated that He II $\lambda 4542$ is also in absorption.

\section{Line profile variability}

As pointed out in Paper I, the He II $\lambda 4686$ emission line in the spectrum of WR 20a displays a prominent profile variability that appears definitely phase-locked. This variability is not restricted to this line, but is also seen in $\mathrm{H} \alpha$. Analysing these modulations should help us to understand the interactions between the two stars.

\subsection{The $\mathrm{H} \alpha$ line}

The variations of the $\mathrm{H} \alpha$ line are illustrated in Fig. 2. At phases near conjunction $(\phi \simeq 0.0$ and $\phi \simeq 0.5$ ), the line displays a rather narrow double-peaked profile with the red peak being somewhat stronger. On the other hand, at phases near quadrature the line appears broader with some indication of more than two peaks. This behaviour suggests the existence of one or several emission components possibly arising from an interaction between the components of WR 20a.

We have measured the equivalent width $(E W)$ of the $\mathrm{H} \alpha$ line integrated between 6499 and $6646 \AA$ as a function of orbital phase. The results are reported in Table 1 . We estimate that the uncertainties affecting our $E W$ measurements are of order $\sim 5 \%$. In Fig. 3, the EWs are compared to the I-band light curve 
Table 1. Journal of our red spectroscopic observations of WR 20a. The first and second columns provide the heliocentric Julian date (in the format HJD-2 450000 ) and the orbital phase computed using the ephemeris of Bonanos et al. (2004). Phase $\phi=0.0$ corresponds to the "primary eclipse" with the slightly less massive star in front. Columns 3 and 4 respectively indicate the grism used for the observation (see text) and the equivalent width of the $\mathrm{H} \alpha$ emission line as integrated between 6499 and $6646 \AA$ (see Sect. 4).

\begin{tabular}{cccc}
\hline \hline Date & $\phi$ & Grism & $\begin{array}{c}E W(\mathrm{H} \alpha) \\
(\AA)\end{array}$ \\
\hline 3032.766 & 0.094 & $\# 6$ & 33.6 \\
3033.762 & 0.364 & $\# 6$ & 28.7 \\
3034.760 & 0.635 & $\# 6$ & 31.4 \\
3036.762 & 0.178 & $\# 6$ & 31.4 \\
3037.784 & 0.456 & $\# 6$ & 35.1 \\
3038.765 & 0.722 & $\# 6$ & 31.9 \\
3039.772 & 0.995 & $\# 6$ & 42.2 \\
3040.760 & 0.263 & $\# 6$ & 31.4 \\
3041.747 & 0.536 & $\# 6$ & 40.6 \\
3042.734 & 0.798 & $\# 6$ & 31.0 \\
3179.472 & 0.895 & $\# 5$ & 26.6 \\
\hline
\end{tabular}

presented by Bonanos et al. (2004). There is a net increase in the apparent line strength by $\sim 40 \%$ during photometric eclipses. Using the $I$-band light curve, we have performed a first order correction of the EWs for the variations of the continuum level. The results are also shown in Fig. 3 by the asterisks and the cross. Apart from a slow modulation with a peak to peak amplitude of about $20 \%$, the corrected $E W$ of the $\mathrm{H} \alpha$ line is almost constant. The remaining modulation might be real and could then reflect partial occultations of the line emitting regions by the stellar bodies. In fact, the minima in the corrected $E W$ s occur about $0.05-0.10$ in phase before the actual photometric eclipses.

In summary, apart from the small modulation described above, the variations of the $E W$ of the $\mathrm{H} \alpha$ line are consistent with a rather constant line flux superposed on a variable continuum level. This suggests that the bulk of the emission arises from an extended region that is only marginally affected by occultations that occur before the actual photometric eclipses.

To interpret the strong profile variability of the $\mathrm{H} \alpha$ line, we have further applied the Doppler tomography technique described by Rauw et al. (2002) to map the line formation region in velocity space (see also e.g. Thaller et al. 2001, for an example of the application of Doppler tomography to early-type binaries). Our method uses a Fourier filtered back projection algorithm (Horne 1991). The radial velocity of any gas flow that is stationary in the binary's rotating frame of reference can be described by a simple relation:

$v(\phi)=-v_{x} \cos (2 \pi \phi)+v_{y} \sin (2 \pi \phi)+v_{z}$

where $\phi$ stands for the orbital phase, whilst $\left(v_{x}, v_{y}, v_{z}\right)$ are the projected velocity coordinates of the gas flow. The above relation assumes that the $x$-axis runs between the stars, from the primary to the secondary, whilst the positive $y$-axis points in the direction of the secondary's orbital motion. The projected $v_{x}$ and $v_{y}$ velocities are related to the absolute velocity coordinates through the relation $\left(v_{x}, v_{y}\right)=\left(V_{x} \sin i, V_{y} \sin i\right)$. Finally,
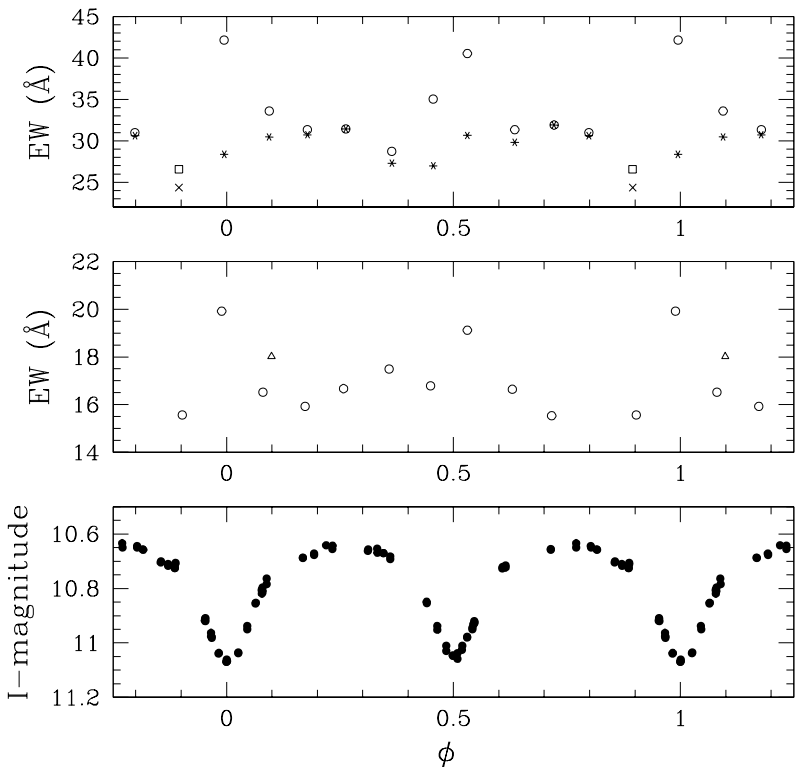

Fig. 3. Upper panel: variations of the measured $E W$ of the $\mathrm{H} \alpha$ emission line between 6499 and $6646 \AA$ (open symbols) and of the $E W \mathrm{~s}$ corrected for the variations of the continuum (asterisks). The open square and the cross correspond respectively to the measured and the corrected $E W$ of the line in the June 2004 spectrum. Middle panel: variations of the measured $E W$ of the He II $\lambda 4686$ line between 4656 and $4723 \AA$ (see text). The triangle refers to the measurement from our 2002 campaign (see text). Lower panel: photometric light curve of WR 20a in the I-band as reported by Bonanos et al. (2004).

the $z$-axis is perpendicular to the orbital plane. The Doppler map consists of a projection of relation (1) on the $\left(v_{x}, v_{y}\right)$ plane. Therefore, for a given value of $v_{z}$, each pixel in a Doppler map, specified by its velocity coordinates is associated with a particular form of Eq. (1) (see e.g. Horne 1991, for a detailed discussion of the method).

To avoid a bias due to the lower resolution of the June 2004 spectrum, we use only the data from the January-February observing run. Our data were weighted so as to account for the uneven sampling of the orbital cycle as well as for the effect of the photometric eclipses. To provide a rough indication of the "point spread function" in velocity space, we have computed the Doppler map of a constant discrete emission feature convolved with the instrumental response and "observed" with the same sampling as the actual data (shown by the insert in Fig. 4).

The $v_{z}$ parameter can be seen as some sort of - a priori unknown - apparent systemic velocity of the $\mathrm{H} \alpha$ line. In Paper I we found a wide range (from -77 to $-18 \mathrm{~km} \mathrm{~s}^{-1}$ ) in apparent systemic velocities for different spectral lines. All the lines studied in Paper I (including the $\mathrm{H} \delta$ and $\mathrm{H} \gamma$ Balmer lines) are in absorption apart from the N IV $\lambda 4058$ emission. In the spectra of WNL stars, the latter line is often considered as the most reliable indicator of the true stellar velocity. Its apparent systemic velocity being $-51 \mathrm{~km} \mathrm{~s}^{-1}$, we have thus adopted $v_{z}=-50 \mathrm{~km} \mathrm{~s}^{-1}$ in the Doppler map (Fig. 4) presented here. We have also carried out our analysis for values of $v_{z}$ in the range -80 to $+20 \mathrm{~km} \mathrm{~s}^{-1}$. While some details of the map change, we find that its general appearance does not depend 


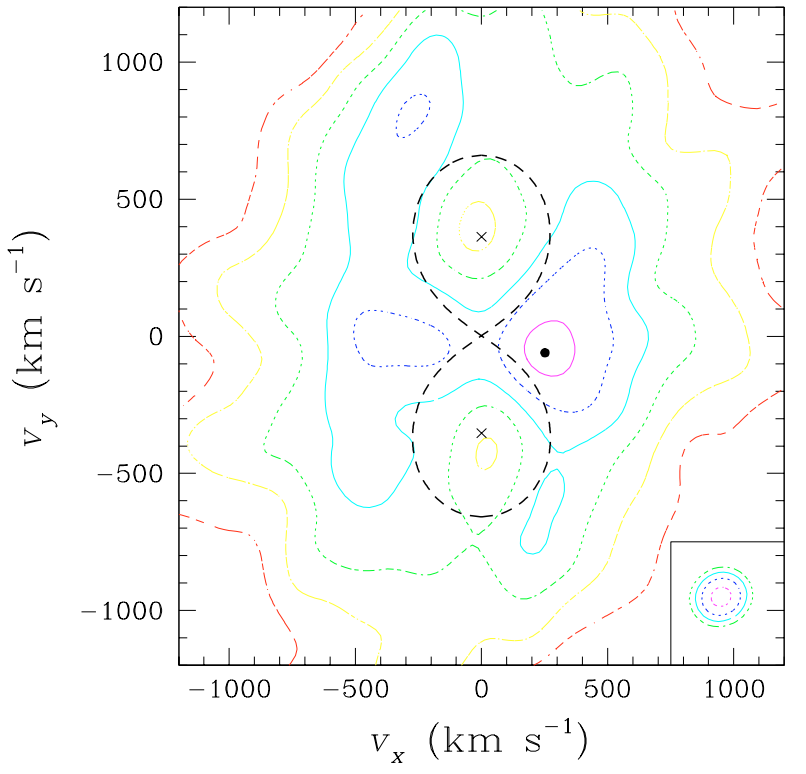

Fig. 4. Doppler map of the $\mathrm{H} \alpha$ emission line in the spectrum of WR 20a. The crosses correspond to the radial velocity amplitudes of the centre of mass of the primary (bottom) and secondary (top) as derived from our orbital solution in Paper I. The shape of the Roche lobe in velocity-space (thick dashed line) has been calculated for a mass ratio of 0.99. The Doppler map was computed with $v_{z}$ set to $-50 \mathrm{~km} \mathrm{~s}^{-1}$. The black dot indicates the position of the highest peak and the contours correspond to levels of $0.95,0.80,0.65,0.50,0.35$ and 0.20 times the maximum emissivity. The insert in the lower right corner illustrates the Doppler map of a constant Gaussian with a FWHM corresponding to the spectral resolution of our data and observed with the same sampling as the actual data. This figure appears in colour in the electronic version of the journal.

on the adopted value of $v_{z}$ within the range of values explored here.

The Doppler map reveals an extended line formation region in velocity space with a primary maximum around $(250,-60)$. A secondary maximum is found near $(-330,-10)$. Thus the maximum emission arises from plasma that moves with a phase shift of almost exactly $\pi / 2$ compared to the orbital motion of the two stars. It should also be stressed that the velocities of the centres of mass of the two stars are actually associated with local minima of the emissivity in the Doppler map.

\subsection{The He II $\lambda 4686$ line}

The analysis of the variations of He II $\lambda 4686$ is less secure than for $\mathrm{H} \alpha$, mainly because of the blends with a broad emission bump on the blue side between 4640 and $4660 \AA$. However, the He II $\lambda 4686$ profile and its variations are extremely similar to those of $\mathrm{H} \alpha$ (see Fig. 2) suggesting that the two lines form in the same physical regions.

The $E W$ of the He II $\lambda 4686$ line, as measured between 4656 and $4723 \AA$, changes as a function of orbital phase. The variations are apparently less pronounced than in the case of $\mathrm{H} \alpha$ (Fig. 3), though they are certainly significant compared to the estimated uncertainties on the measurements of $\sim 5-10 \%$. We caution however that the impression of more erratic variations

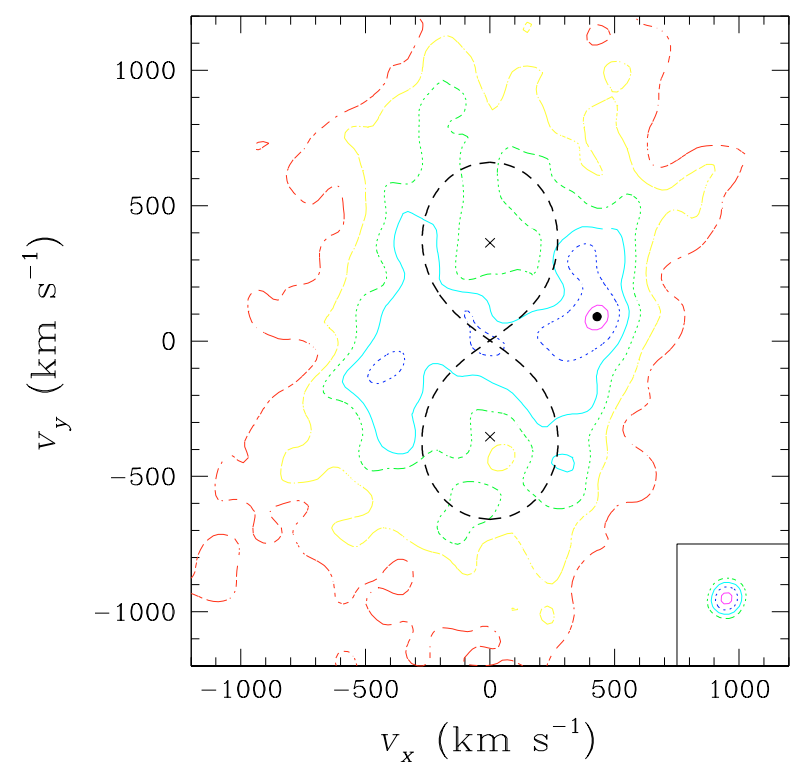

Fig. 5. Same as Fig. 4, but for the He II $\lambda 4686$ emission.

is to a large extent due to the point at phase 0.099 (the triangle in Fig. 3), which comes from our 2002 campaign (see Paper I). Extrapolating the photometric ephemeris back to the epoch of this observation could result in rather large uncertainties on the orbital phase. If we omit this point, the variations of the $E W$ are quite reminiscent of those of the $\mathrm{H} \alpha$ line. Note that we have chosen not to correct the observed $E W \mathrm{~s}$ for the modulation of the continuum level since the light curve in the $I$-band filter might not be representative of the actual variations of the continuum in the blue spectral range.

Finally, the Doppler map of this line (Fig. 5) is again quite similar to the one of $\mathrm{H} \alpha$. To avoid too severe contamination of the Doppler map by the emission bump bluewards of He II $\lambda$ 4686, we have restricted the velocity interval in the back projection to $[-2000,2000] \mathrm{km} \mathrm{s}^{-1}$. The highest peaks (at about $(425,90)$ and $(-400,-95))$ are again found at a phase shift of almost $\pi / 2$ compared to the orbital motion.

\section{Fundamental parameters}

Adopting the orbital period of 3.686 days as derived by Bonanos et al. (2004), we have recalculated an orbital solution $^{2}$ using our data from Paper I (with the same weights as in Paper I, but reversing the primary/secondary identifications for the data of HJD 2452355.6$)$. This new solution yields $K_{1}=362.5 \pm 9.0 \mathrm{~km} \mathrm{~s}^{-1}, K_{2}=365.6 \pm 9.1 \mathrm{~km} \mathrm{~s}^{-1}$, $\gamma_{1}=20.1 \pm 5.8 \mathrm{~km} \mathrm{~s}^{-1}, \gamma_{2}=-5.2 \pm 5.9 \mathrm{~km} \mathrm{~s}^{-1},\left(a_{1}+a_{2}\right) \sin i=$ $53.0 \pm 0.7 R_{\odot}, m_{1} \sin ^{3} i=74.0 \pm 4.2 M_{\odot}$ and $m_{2} \sin ^{3} i=$ $73.3 \pm 4.2 M_{\odot}{ }^{3}$. Combining these results with the orbital inclination $\left(74.5^{\circ} \pm 2.0^{\circ}\right)$ inferred by Bonanos et al. yields absolute masses of 82.7 and $81.9 M_{\odot}$ for the primary and secondary respectively.

\footnotetext{
${ }^{2}$ Bonanos et al. already recalculated an orbital solution, adopting a priori a single value for the systemic velocity of both stars. Since the lines measured to evaluate the radial velocities form in moving stellar winds, this could in principle bias the amplitude of the orbital solution.

${ }^{3}$ All error bars in this paper correspond to $1 \sigma$ uncertainties.
} 
The light curve solution of Bonanos et al. provides also some constraints on the stellar radii. These authors found that the surfaces of the stars are described by a Roche potential of $\Omega=3.92$, which corresponds to heavily deformed objects with average radii of $\sim 19.7 \pm 0.3 R_{\odot}$. Wolf-Rayet stars typically have optically thick stellar winds, such that their stellar radii and corresponding temperatures are generally quoted at Rosseland optical depth of order 10 instead of the usual 2/3. Therefore, since the light curve model of Bonanos et al. does not account for an extended atmosphere, it is not clear a priori whether these observational values refer to the hydrostatic radius or to some specific value of the optical depth. However, in the case of WR 20a, the wind densities of the WN6ha components are only slightly larger than for typical $\mathrm{O}$ supergiants, such that their radii at Rosseland optical depths $\tau=2 / 3$ and $\tau=10$ differ by less than $0.1 R_{\odot}$.

Bonanos et al. (2004) argue that the $I$-band light curve of WR 20a indicates a slightly deeper primary eclipse and they attribute this to a lower effective temperature $(40300 \mathrm{~K})$ for the secondary compared to the primary $(42000 \mathrm{~K})$. From the spectroscopic point of view, the primary and secondary cannot be distinguished (see Paper I). While it is true that a small difference in effective temperature (or stellar radius) may not necessarily be detectable in the spectrum, we caution that the difference in depth of the eclipses in the light curve of Bonanos et al. could also be due to the scatter of the data around the light curve solution. New photometric observations, especially including additional filters are needed to confirm the difference in eclipse depth and discriminate between a difference in temperature or other model parameters such as the radii. Therefore, in the following, we consider that both stars have identical properties.

To determine the stellar properties of WR 20a we have utilized the non-LTE code of Hillier \& Miller (1998) which solves the radiative transfer equation subject to the constraints of statistical and radiative equilibrium, in a spherical, extended atmosphere. Line blanketing is incorporated directly through the use of a super-level approach. We use a similar atomic model to that employed by Walborn et al. (2004) in their study of earlytype $\mathrm{O}$ stars, including H I, He I-II, C III-IV, N III-V, O III-VI, Ne III-V, Si IV, P V, S IV-VI, Ar III-VI, Fe V-VII and Ni V-VII (see Walborn et al. for further details).

The velocity in the supersonic part of the wind is described by a standard $\beta$-law

$$
v(r)=v_{\infty}\left(1-\frac{R_{*}}{r}\right)^{\beta}
$$

with $\beta=1$. Since no ultraviolet spectra of WR 20a are available, we adopt a representative terminal wind velocity of $v_{\infty}=$ $2800 \mathrm{~km} \mathrm{~s}^{-1}$. While this value is larger than for other Galactic WN6ha stars (Crowther \& Dessart 1998), we note that lower values of $v_{\infty}$ would result in a poorer match of the He II $\lambda 4686$ profile. We further adopted $v_{\mathrm{eq}} \sin i=200 \mathrm{~km} \mathrm{~s}^{-1}$ for the line fits.

For stars such as the WN6ha components in WR 20a, He I lines are weak or absent and we are hence forced to use the nitrogen lines to establish the ionization balance (as in
Walborn et al. 2004). There is no obvious indication of an enriched He abundance in the spectrum of WR 20a. On the other hand, it is extremely difficult to determine accurate $\mathrm{He} / \mathrm{H}$ abundances for extreme stars such as the WN6ha components in WR 20a. Therefore, we have adopted a He/H number abundance ratio of 0.1 .

The mass-loss rate $\left(8.5 \times 10^{-6} M_{\odot} \mathrm{yr}^{-1}\right)$ was determined from the strength of the $\mathrm{H} \alpha$ line assuming a clumped wind with a volume filling factor $f=0.1$. The $\mathrm{H} \alpha$ line forming region in our model peaks at about $1.4 R_{*}$, but extends to beyond $10 R_{*}$. If the wind volume filling factor in this region were different, the actual $\dot{M}$ would scale roughly as $\sqrt{f}$. For instance, a smooth wind model would require a mass-loss rate of $2.5 \times 10^{-5} M_{\odot} \mathrm{yr}^{-1}$ to match the $\mathrm{H} \alpha$ and He II $\lambda 4686$ lines. Given the extent of the line formation region, it seems likely that the extra emission from the wind interaction zone contributes only a moderate fraction (10 to 20 per cent) of the total $\mathrm{H} \alpha$ emission. Finally, we note that the spectra we have fitted are in principle the least affected by the emission from the wind interaction zone, but were taken at phases affected by the photometric eclipse. Therefore, the equivalent width of the $\mathrm{H} \alpha$ line could be overestimated by $\sim 15 \%$, implying a reduction of $\dot{M}$ of order $10 \%$.

For $T_{\text {eff }}=43000 \pm 2000 \mathrm{~K}$, we find an overall agreement for the nitrogen lines: weak P-Cygni lines in N v, strong emission in N IV and weak emission in N III (see Fig. 6). The nitrogen abundance is found to be $0.5 \%$ (by mass), i.e. six times solar. The C IV 5801, 5812 lines can in principle be used to constrain the carbon abundance although these lines are rather sensitive to the extreme UV flux distribution. As stated above, the equivalent width of this blend on our spectra is about $1.0 \AA$. For an assumed carbon abundance of 0.1 (respectively 0.04) times solar, our model yields an equivalent width of $4.8 \AA$ (respectively $2 \AA$ ), too large compared to the observed value. While the lack of other diagnostics prevents us from deriving a more precise abundance, we conclude nevertheless that $\mathrm{C}$ is severely depleted in the components of WR 20a. For oxygen, there is no good diagnostic line in the optical and we thus cannot constrain its abundance with our present data set.

The parameters of our best model are given in Table 2. Note that the value of the gravity does not directly follow from the model atmosphere code (which uses a crude treatment of the photospheric structure at depth), but stems from the binary mass determination and the stellar parameters derived from our model. To get a first approximation of $L_{\mathrm{bol}}$, we either adopted $R_{*}$ from the light curve solution of Bonanos et al. (2004) or $M_{V}=-6.5$ (see also Sect. 6.2). Both assumptions consistently yield $\log L_{\mathrm{bol}} / L_{\odot} \simeq 6.0$. The number of Lyman ionising photons produced by each of the WN6ha stars in WR 20a would be $Q_{0}=7.2 \times 10^{49} \mathrm{~s}^{-1}$.

Our derived temperature provides a bolometric correction of $-3.91 \mathrm{mag}$. This value is somewhat larger than the mean bolometric correction of $-3.70 \pm 0.08$ derived from the analysis of five WN6ha stars by Crowther \& Dessart (1998). The latter value was based on lower temperature unblanketed models and a re-analysis of the WN6ha stars in NGC 3603 and R 136 with contemporary models might therefore lead to bolometric corrections comparable to that of WR 20a. 
Table 2. Fundamental parameters of the WN6ha stars of WR 20a and of their stellar winds as determined from our spectral analysis. Note that the luminosity and mass-loss rate are given for a single component. The mass loss rate is based on a clumped wind model with a volume filling factor of $f=0.1$.

\begin{tabular}{lc}
\hline \hline \multicolumn{1}{c}{ Parameter } & Value \\
\hline Mass $\left(M_{\odot}\right)$ & $82.7 \pm 5.5$ (primary) \\
Mass $\left(M_{\odot}\right)$ & $81.9 \pm 5.5$ (secondary) \\
$L_{\text {bol }}\left(L_{\odot}\right)$ & $(1.15 \pm 0.15) \times 10^{6}$ \\
$T_{\text {eff }}(\mathrm{K})$ & $43000 \pm 2000$ \\
$\dot{M}\left(M_{\odot} \mathrm{yr}^{-1}\right)$ & $8.5 \times 10^{-6}$ \\
$R_{*}\left(R_{\odot}\right)$ & $19.3 \pm 0.5$ \\
$v_{\infty}\left(\mathrm{km} \mathrm{s}^{-1}\right)$ & 2800 (adopted) \\
$\log g$ & 3.8 \\
$(B-V)_{0}$ & -0.33 \\
$(V-I)_{0}$ & -0.36 \\
$E(B-V)$ & $1.94 \pm 0.05$ \\
$A_{\mathrm{\vee}}(\mathrm{mag})$ & $6.02 \pm 0.16$ \\
$d(\mathrm{kpc})$ & $7.9 \pm 0.6$ \\
\hline
\end{tabular}

\section{Discussion and future perspectives}

\subsection{Wind interactions in WR20a}

\subsubsection{The optical emission lines}

The $\mathrm{H} \alpha$ Doppler map and the profile variations are reminiscent of those of the O-star binary HD 149404 (Thaller et al. 2001; Rauw et al. 2001) and we can interpret this as a signature of an interaction between the winds of the two stars. In the case of WR 20a, both stars have roughly identical spectral types (see Paper I) and should thus have winds of similar strengths. In such a case, we expect the wind interaction zone to be roughly symmetrical about the centre of mass of the binary system ${ }^{4}$.

First of all, we stress that part of the profile variations of $\mathrm{H} \alpha$ and He II $\lambda 4686$ is most probably simply due to the loss of spherical symmetry of the winds in this binary system. For instance, it is likely that the properties of the stellar winds along the binary axis between the two stars deviate from those of the winds along other directions. Effects such as "wind focusing" and "radiative inhibition" (Stevens 1988; Stevens \& Pollock 1994) are likely to impact on the mass-loss between the stars. Following the formalism of Stevens \& Pollock, one would expect that for a binary consisting of two stars of equal mass and equal luminosity, the mass-loss rate per unit solid angle along the binary axis (the so-called stagnation mass-loss rate) might be reduced compared to the corresponding single star massloss rate. In fact, in this case, the inhibition effect of the companion's radiation field should dominate over the effect of its gravitational field. However, it must be stressed that the radiative flux from each star reflects off the surface of its companion in radiative equilibrium and as a result of this effect, the actual mass loss might essentially be unchanged by inhibition (Gayley et al. 1999). On the other hand, it is likely that the stagnation wind velocity law differs from that along other directions and

\footnotetext{
${ }^{4}$ Given the lack of information on magnetic fields of early-type stars, we do not consider the impact of magnetic fields on the interaction in WR 20a.
}

the winds should reach only moderate velocities before they collide. Finally, the "radiative braking" mechanism described by Gayley et al. (1997) only occurs in binaries with strongly unequal wind strengths and should thus not play a significant role in the wind interaction region of WR 20a.

In Fig. 7, we provide a very schematic view of the possible wind interaction in WR 20a. In this figure, we have plotted the stars with their shape corresponding to a Roche potential $\Omega=3.92$ as found in the light curve solution of Bonanos et al. (2004). It is obvious that, due to the proximity of the stars in the binary system and to the radiative inhibition mechanism, the winds do not have the time to accelerate to large velocities before they collide. In the absence of orbital motion, we would expect the wind contact surface to be roughly planar. Given the large orbital velocities and the comparatively low pre-shock wind velocity (both of order $500 \mathrm{~km} \mathrm{~s}^{-1}$ ), we expect the wind interaction zone to be heavily skewed by the Coriolis force instead. To first order, we can estimate the deflection angle to be $\psi=\arctan \left(v_{\text {orb }} / v_{\text {wind }}\right) \simeq \pi / 4$.

Both the Doppler tomography and the variations of the equivalent widths provide constraints on the possible location of the formation regions of the $\mathrm{H} \alpha$ and $\mathrm{He}$ II $\lambda 4686$ emission peaks. The fact that the line emitting region might be partially occulted at phases of about $0.05-0.1$ before the actual conjunctions (see Sect. 4 above) suggests that the $\mathrm{H} \alpha$ emission peaks arise in plasma that is located in two regions that are shifted by $\sim 20-35^{\circ}$ from the binary axis. Since we do not observe similar occultations at phases $0.05-0.1$ after conjunction, the line emitting region must be rather elongated (as illustrated in Fig. 7) such that the strongest occultation occurs at these phases. The $\left(v_{x}, v_{y}\right)$ coordinates in the Doppler map are then a combination of the orbital motion and the bulk motion (tangential to the shock) of the post-shock plasma. While it is clear that a detailed modelling of the wind interaction in WR 20a is needed to confirm (or reject) our scenario, we tentatively suggest that the wind-wind collision produces a high density zone where the plasma undergoes a very efficient cooling, thus favouring the formation of excess optical emission lines (such as the peaks seen in $\mathrm{H} \alpha$ or $\mathrm{He}$ II $\lambda$ 4686) through recombination. A detailed model of the wind interaction in WR 20a (accounting for the orbital motion and the efficient radiative cooling of the post-shock plasma) should tell us where in the interaction zone the X-ray emission arises and how this impacts on the formation of optical recombination lines. A weakness of our simple model is that it suggests that the material that produces the red-shifted emission peak should be occulted whilst the blue-shifted emission peak should not undergo occultations by the stellar bodies. This seems at odds with the fact that the red peak is usually the strongest one (see Fig. 2). The latter feature clearly calls for sophisticated hydrodynamic simulations including optical line emission diagnostics as well as new observations that should describe the orbital variations of the two peaks with a better phase resolution.

Alternatively, one could also imagine that the $\mathrm{H} \alpha$ and He II $\lambda 4686$ line emissions arise in jets impacting on the rear sides of the stars. For instance, Lubow \& Shu (1975) have shown that in a RLOF situation, material flowing from the inner Lagrangian point should impact on the rear side of the 

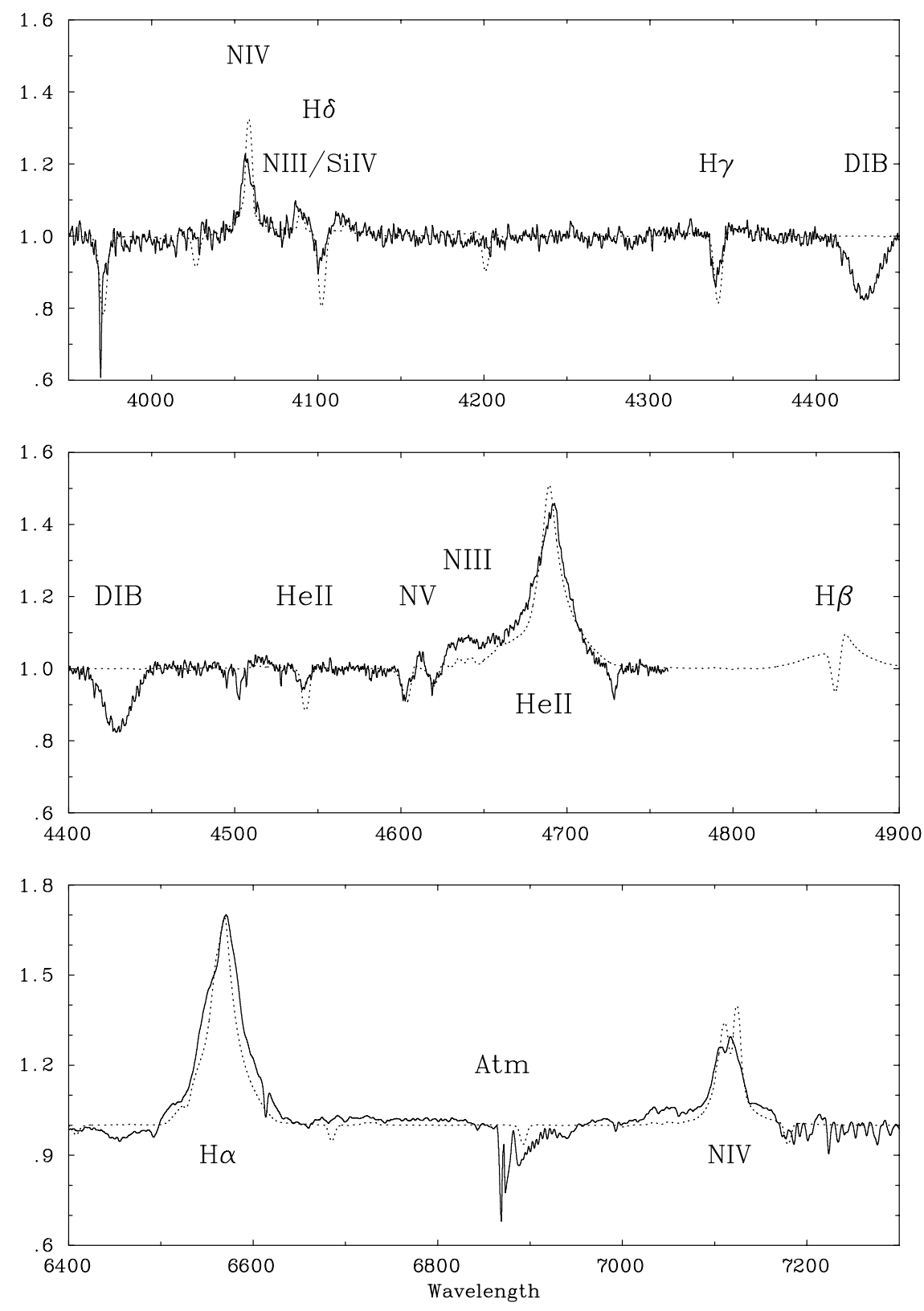

Fig. 6. Comparison between the observed spectrum at phase $\sim 0.46$ (solid line) and the predictions from our best atmospheric model (dashed line).

companion star. However, in WR 20a, the light curve solution suggests that both stars are still within their Roche lobe. Moreover, it is not clear how one could explain the existence of two such jets (one moving from the primary to the secondary and the second one in the opposite direction) in such a scenario.

\subsubsection{X-ray emission from WR20a}

Wind-wind collisions in early-type binaries are commonly believed to produce an excess X-ray emission (compared to the total intrinsic X-ray emission from the two stars) arising in the hot shocked plasma between the stars (e.g. Stevens et al. 1992).

Little is known so far about the X-ray emission of WR 20a: Pollock et al. (1995) reported a $4 \sigma$ detection during the ROSAT All Sky Survey with a PSPC count rate of
$(5.8 \pm 1.4) \times 10^{-2}$ cts s$^{-1}$, whilst diffuse X-ray emissions from the HII region RCW 49 and from the stars of Westerlund 2 were noted by Mereghetti \& Belloni (1995). Apparent diffuse emission from this cluster was previously also reported based on EINSTEIN-IPC observations (Goldwurm et al. 1987). Goldwurm et al. attributed this feature either to a wind-blown bubble or a supernova remnant.

We have checked the ROSAT archive for pointed observations of WR 20a and we found two HRI and one PSPC data sets. The PSPC observation (rp400329n00) corresponds to the data analysed by Mereghetti \& Belloni (1995). Only one of the HRI pointings (rh201611a01) has a sufficient duration (18 ks) to provide useful information. The HRI image (see Fig. 8) indicates that WR 20a is detected with a count rate of $(2.3 \pm 0.5) \times 10^{-3}$ cts s$^{-1}$. However, this image also reveals a 


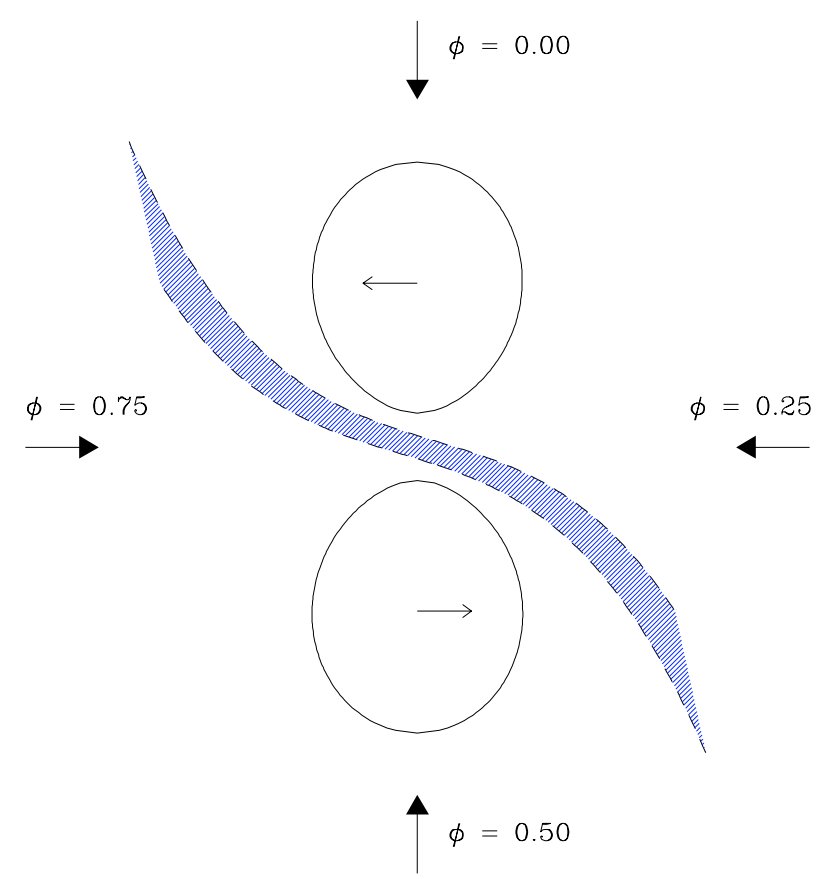

Fig. 7. Schematic view of a possible wind interaction process in WR 20a. The direction of the line of sight is projected on the orbital plane for the phases of quadrature and conjunction. The peaks of the $\mathrm{H} \alpha$ and He II $\lambda 4686$ emissions arise in the shaded area, though the full emitting region could extend beyond the limits of our figure.

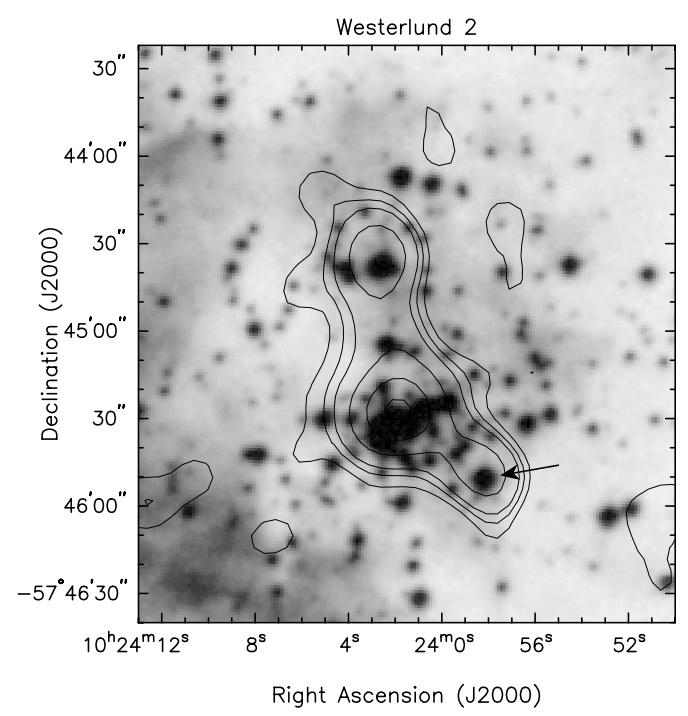

Fig. 8. ROSAT-HRI X-ray contours overlaid on a DSS image of the Westerlund 2 cluster. The X-ray emission associated with WR 20a (10h23min $58 \mathrm{~s},-57^{\circ} 45^{\prime} 49^{\prime \prime}, \mathrm{J} 2000$; indicated by the arrow) is clearly seen. The bright X-ray source to the north of the cluster core appears to be most likely associated with star No. $18(\mathrm{O} 7: \mathrm{V})$ of Moffat et al. (1991).

somewhat brighter source, associated with the core of Westerlund 2, and located at $\sim 40 "$ North-East from WR 20a. While the two sources can be almost separated with the HRI, they are not resolved with the PSPC (as becomes also clear from the elongated shape of the PSPC source) and the count rate reported by Pollock et al. (1995) is therefore most likely due to the combined X-ray emission from Westerlund 2 and WR 20a (see also Mereghetti \& Belloni 1996).

The X-ray source associated with the cluster core is responsible for most of the apparently diffuse emission discussed by Goldwurm et al. (1987). We note also that star No. 18 of Moffat et al. (1991) is apparently associated with an X-ray source that might be significantly brighter than what would be expected based on the spectral type of this star $(\mathrm{O} 7: \mathrm{V})$ and on the canonical relation between X-ray and bolometric luminosity (see Berghöfer et al. 1997). This could indicate that star no. 18 is actually a colliding wind binary.

Westerlund 2 was recently observed with the ACIS-I instrument onboard Chandra. These observations confirm indeed the existence of a diffuse X-ray emission (Townsley et al. 2004) and allow for the first time the study of many point sources that were previously not resolved. Tsujimoto et al. (2004) report a fit of the ACIS-I X-ray spectrum of WR 20a using an optically thin thermal plasma model with $k T=2.1 \mathrm{keV}$ and a neutral hydrogen column density of $2.1 \times 10^{22} \mathrm{~cm}^{-2}$. The latter value is about twice as large as the interstellar neutral hydrogen column density $\left(N_{\mathrm{H}}=1.1 \times 10^{22} \mathrm{~cm}^{-2}\right)$ evaluated from the $E(B-V)$ value determined in Sect. 6.2. This feature is not unusual for Wolf-Rayet stars: extra X-ray absorption is produced by the material in the stellar wind and is observed for instance in the case of the WN6ha star WR 25 (Raassen et al. 2003). Even worse, in the case of the WN8 star WR 40, the wind absorption is probably so large that no $\mathrm{X}$-ray emission is actually escaping (Gosset et al. 2005). The best-fit plasma temperature found by Tsujimoto et al. (2004) is rather high for early-type stars, which could be a signature of the colliding wind interaction. Correcting for the entire hydrogen column density, Tsujimoto et al. infer an unabsorbed flux of $\left(1.1_{-0.3}^{+0.9}\right) \times 10^{-12} \mathrm{erg} \mathrm{cm}^{-2} \mathrm{~s}^{-1}$ in the $0.9-8 \mathrm{keV}$ energy range. Unfortunately WR 20a fell rather close to a gap of the ACISI detector in the Chandra observation, which complicates the analysis (Tsujimoto et al. 2004). If instead of correcting for the entire column density we correct only for the absorption of the ISM material, we infer an X-ray flux of $6.3 \times$ $10^{-13} \mathrm{erg} \mathrm{cm}^{-2} \mathrm{~s}^{-1}$ in the $0.5-8 \mathrm{keV}$ energy range.

Turning back to the ROSAT observations, we can use the Chandra spectral parameters to convert the HRI count rate into observed and ISM-corrected X-ray fluxes. Doing so, we estimate an ISM corrected X-ray flux of $1.0 \times 10^{-12} \mathrm{erg} \mathrm{cm}^{-2} \mathrm{~s}^{-1}$ in the $0.5-8 \mathrm{keV}$ energy band. This number is about 1.5 times larger than the Chandra result, though it is still within the rather large error bars of the latter. This difference might be either attributable to the uncertainties in the flux determinations or to actual variations of the X-ray flux from WR 20a. For a distance of $7.9 \mathrm{kpc}$, we derive an X-ray luminosity of $7.5 \times 10^{33} \mathrm{erg} \mathrm{s}^{-1}$ from the ROSAT-HRI result. With a total bolometric luminosity of $2.3 \times 10^{6} L_{\odot}$ (see Sect. 5), this yields $\log \left(L_{\mathrm{X}} / L_{\text {bol }}\right)=-6.07$.

Comparing these results to the luminosities of presumably single WN stars (Wessolowski 1996), we find that the X-ray luminosity of WR 20a, although not exceptionally large, is clearly among the highest values of the sample. Moffat et al. (2002) reported X-ray luminosities of three WN6ha stars in NGC 3603 as observed with ACIS-I. For NGC 3603 stars A1, B and C, these authors obtain $>10^{34}, 2-3 \times 10^{33}$ 
and $>4 \times 10^{34} \mathrm{erg} \mathrm{s}^{-1}$ respectively (the lower limits for stars $\mathrm{A} 1$ and $\mathrm{C}$ are due to pile-up in the Chandra data). Although it remains to be established what fraction of their X-ray fluxes arises from colliding wind interactions, we note that the two X-ray brightest Wolf-Rayet objects in NGC 3603 are either confirmed or suspected binary systems (see the discussion in Moffat et al. 2002). Since the wind properties of the components of WR 20a are more typical of extreme Of stars than of classical Wolf-Rayet stars, we compare the observed X-ray luminosity of WR 20a also to the empirical $L_{X}-L_{\text {bol }}$ relation for OB stars derived by Berghöfer et al. (1997). The Berghöfer et al. relation applies to the X-ray luminosity in the $0.1-2.0 \mathrm{keV}$ energy range. Over this energy band, our estimate of the ISM absorption corrected X-ray flux amounts to $2.9 \times 10^{-13} \mathrm{erg} \mathrm{cm}^{-2} \mathrm{~s}^{-1}$ and the corresponding X-ray luminosity is only about $30 \%$ larger than the value expected from the Berghöfer et al. $L_{X}-L_{\text {bol }}$ relation. This is well within the intrinsic scatter of the latter relation, thus suggesting that there is only little if any excess emission in the ROSAT band. However, only additional X-ray observations with Chandra combining spectral information and a high spatial resolution and monitoring the possible variations of the X-ray flux over the full orbital cycle will allow us to check the existence of an excess X-ray emission and of a possible modulation of the X-ray flux.

Finally, we can compare our estimated $L_{X}$ to the expected $\mathrm{X}$-ray luminosity from a colliding wind binary with equal winds (e.g. Pittard \& Stevens 2002, and references therein). Adopting the $\beta$ velocity law from Sect.5, we estimate $v \simeq$ $500 \mathrm{~km} \mathrm{~s}^{-1}$ at the stagnation point of the wind-wind collision. This implies $L_{\mathrm{X}}^{\text {theor }} \simeq 2.2 \times 10^{35} \mathrm{erg} \mathrm{s}^{-1}$ which is about a factor 30 larger than the observed $L_{\mathrm{X}}$. Therefore, the actual X-ray luminosity must be much smaller than the theoretical prediction. Again, radiative inhibition (Stevens \& Pollock 1994) could play a key role here. This effect could indeed lower the pre-shock velocity substantially, thereby reducing the expected $\mathrm{X}$-ray luminosity of the wind interaction zone.

\subsection{The relationship between WR20a and Westerlund 2}

In Paper I we pointed out that, mainly because of the possibility of eclipses, the existing photometric data did not allow us to draw a firm conclusion on the relationship between WR 20a and the open cluster Westerlund 2. With the new information available on WR 20a, we can now readdress this issue.

\subsubsection{The reddening towards WR20a}

Moffat et al. (1991) gathered $U B V$ CCD photometry of Westerlund 2, thereby discovering the high differential reddening towards this cluster. Following their Fig. 12, WR 20a is apparently located in a region of high extinction $(E(B-V) \geq 1.80)$.

Unfortunately, most photometric data of WR 20a quoted in the literature are of little use to constrain the distance and reddening of this system. As a matter of fact, in most cases, the orbital phase at which the observations were obtained is unknown and the data could thus be affected by the eclipses. Even worse, some of these observations were apparently obtained on different nights (i.e. at different phases). The latter limitation does not hold, however, for the study of Bonanos et al. (2004) and for the most recent photometric study of Westerlund 2 by Carraro $\&$ Munari (2004). Bonanos et al. measured $V=13.45$ and $V-I=2.80$ during maximum light. From the analysis of the WR 20a spectrum in Sect. 5, we find $(V-I)_{0}=-0.36$, which is close to the intrinsic $V-I$ colour index of $\mathrm{O}$ supergiants $(-0.39$ to -0.41 , see Clark et al. 2004). With $E(V-I)=3.16$ and using the reddening law of Savage \& Mathis (1979), we estimate $E(B-V)=1.98$ and $A_{\mathrm{V}}=6.12 \mathrm{mag}$.

Carraro \& Munari (2004) obtained their UBVRI photometry of Westerlund 2 during a single night in June 1992. They found that the $E(B-V)$ colour excess varies between 1.5 and 2.1 across the field of view of the cluster. According to the complete photometric data provided by Carraro (2004), WR 20a had $V=13.28 \pm 0.05, B-V=1.63 \pm 0.04$, $U-B=0.29 \pm 0.04$ and $V-I=2.52 \pm 0.04$. Quite surprisingly, the $V$ magnitude of Carraro \& Munari is slightly brighter $(\Delta V=0.17)$ than the magnitude at maximum light determined by Bonanos et al. (2004). Also, the $V-I$ index determined by Carraro \& Munari is significantly lower than the colour quoted by Bonanos et al. Therefore, if we adopt $V-I=2.52$, we find $E(B-V)=1.80$ with the above technique. Alternatively, from our non-LTE analysis in Sect. 5 we have found that $(B-V)_{0}=-0.33$ and the $B-V$ colour of Carraro \& Munari then results in $E(B-V)=1.96 \pm 0.04$ and $A_{\mathrm{V}}=(6.08 \pm 0.14) \mathrm{mag}$.

A completely independent estimate of the reddening can be obtained from our flux-calibrated spectrum of WR 20a. In fact, Morris et al. (1993) showed that the intrinsic continuum energy distribution of Wolf-Rayet stars in the $1500 \AA-1 \mu \mathrm{m}$ range can be approximated by a power law relation $F_{\lambda}^{0} \sim \lambda^{-\alpha}$ with $\alpha=2.87 \pm 0.38$ for Galactic WNL stars. We have dereddened our flux-calibrated spectrum of WR 20a using the interstellar reddening law of Savage \& Mathis (1979) for different values of $E(B-V)$ with a step of 0.02 in $E(B-V)$. We have then fitted the continuum between 4100 and $6800 \AA$ of this dereddened energy distribution with a power law. In this way, we find that the slope is in agreement with the results of Morris et al. (1993) for $E(B-V)=1.89 \pm 0.11$. The latter value of the colour excess is in very good agreement with the value inferred from the photometric data. Morris et al. (1993) also provide a tight relation between the $(b-v)_{0}$ colour index ${ }^{5}$ and $\alpha$ which indicates that the slope for a WN6ha star could be as steep as $\alpha=3.33$. Such a large $\alpha$ would shift the best fit $E(B-V)$ value towards the upper end of the confidence range.

In summary, the three reddening estimates that are in closest agreement with each other yield $E(B-V)=1.94 \pm 0.05$ and $A_{\mathrm{V}}=(6.02 \pm 0.16) \mathrm{mag}$.

\footnotetext{
5 The $(b-v)_{0}$ index refers to the Smith (1968) ubvr photometric system. This system was designed for Wolf-Rayet stars and is based on a set of essentially emission line-free narrow band filters. The intrinsic $(b-v)_{0}$ colour index of five WN6ha stars studied by Crowther $\&$ Dessart (1998) amounts to $-0.29 \pm 0.02$.
} 


\subsubsection{The distance}

Let us first consider the distance of Westerlund2. Several (somewhat) conflicting determinations of the cluster distance exist in the literature. Moffat et al. (1991) derived a distance of 7.9 ${ }_{-1.0}^{+1.2} \mathrm{kpc}$ for this cluster. Piatti et al. (1998) obtained integrated spectroscopy of Westerlund 2 and arrived at a distance of $5.7 \pm 0.3 \mathrm{kpc}$ and an age of 2-3 Myr. Most recently, Carraro \& Munari (2004) analysed UBVRI photometry of the cluster. Comparing their colour-magnitude diagrams to isochrones from the Padova group, they inferred a distance of $6.4 \pm 0.4 \mathrm{kpc}$ and an age $\leq 2 \mathrm{Myr}$. We note also that distances between 2.3 and $5.8 \mathrm{kpc}$ have been inferred for the RCW $49 \mathrm{H}$ II region either based on ionization or kinematic arguments (see the discussion by Churchwell et al. 2004).

We can now compare this to the distance of WR 20a. In the following we shall use the photometric data of Carraro \& Munari (2004) assuming that they represent the colours and magnitudes of WR 20a at maximum light. From our model atmosphere in Sect. 5, we derive a bolometric magnitude of -10.40 for each component and a bolometric correction of -3.91 . The absolute $M_{V}$ magnitude of each component of WR 20a would thus be $-6.49 \pm 0.08$, in reasonable agreement with a mean $M_{v}=-6.5$ for WN6ha stars (van der Hucht 2001, Table 27) ${ }^{6}$. With $A_{\mathrm{V}}=6.02 \pm 0.16$ derived hereabove and $V=13.28 \pm 0.05$, we arrive at a distance modulus $D M=$ $14.50 \pm 0.17(d=(7.9 \pm 0.6) \mathrm{kpc})$ for the WR 20a system. While this result is in excellent agreement with the cluster distance as determined by Moffat et al. (1991), our distance of WR 20a is significantly larger than the cluster distance of Carraro \& Munari (2004). We caution that the result of Carraro $\&$ Munari could be somewhat biased towards shorter distances if a significant fraction of the brightest stars in Westerlund 2 were binary systems.

In conclusion, we find that our distance determination of WR 20a is in reasonable agreement with (some) other distance estimates of the cluster, supporting the idea that the binary is a probable member of the cluster.

\subsubsection{WR20a as a member of Westerlund 2}

An important issue is the formation of a very massive system like WR 20a in a cluster like Westerlund 2. Though the formation of massive stars still remains an essentially unsolved problem, many observations support the idea that most (if not all) massive stars form in clusters. In very young open clusters such as NGC 6231 and IC 1805, the most massive stars appear to be concentrated in the cluster core. The observed mass segregation in these very young objects is probably not the result of the dynamical evolution of the clusters but seems rather related to the formation process of their most massive members (Raboud \& Mermilliod 1998). A possible explanation for this situation involves a massive star formation scenario that combines competitive gas accretion and stellar collisions in the

\footnotetext{
6 The latter result refers again to the Smith (1968) ubvr photometric system. We have corrected the absolute magnitude given in Table 27 of van der Hucht (2001) for the revised distance of NGC 3603 by Sagar et al. (2001) compared to Crowther \& Dessart (1998).
}

very dense core of the cluster (Bonnell et al. 1998; Portegies Zwart et al. 1999; Bonnell \& Bate 2002). The massive stars formed in this way are expected to be often found in a close binary system. At first sight, this scenario could apply to WR 20a. However, WR 20a is actually located slightly outside the core of Westerlund 2. According to the study of Moffat et al. (1991), the core of the Westerlund 2 cluster consists of a rather compact group containing at least five O6-7:V stars within a radius of 15 arcsec. WR 20a appears to be rather isolated, lying at about 30 arcsec from the centre of the cluster. At a distance of $7.9 \mathrm{kpc}$, this corresponds to a projected distance of $\sim 1.1 \mathrm{pc}$. In this context, we note that the Two Micron All Sky Survey point source catalogue (2MASS, Skrutskie et al. 1997) does not reveal any concentration of sources around WR 20a that could hint towards a heavily obscured group of (proto-) stars.

The position of WR 20a within Westerlund 2 could in principle be explained by the fact that turbulence in a molecular cloud can lead to several sites of massive star formation (Bonnell et al. 2004). Nevertheless, it is surprising that the most massive pair known in Westerlund 2 is not found in the cluster centre and that the cluster core apparently does not contain stars earlier than - and hence more massive than - spectral type O6. Clearly, our knowledge of the stellar population of Westerlund 2 is still too fragmentary. Future studies of this cluster should aim at a determination of the spectral types of the stars in the core as well as of their multiplicity. Also, it would be important to look for a possible population of low-mass premain sequence stars that could provide further insight into the star formation history of this cluster.

An alternative possibility would be that WR 20a actually formed in the cluster core, but got ejected through dynamical interactions (Bonnell 2004). In fact, $N$-body simulations of typical young clusters indicate that they can produce dynamically ejected runaways as a result of encounters involving hard binaries (i.e. binaries with binding energies that exceed the cluster binding energy by a significant factor, Leonard \& Duncan 1990). Depending on the details of the collision, the outcome of such an event can be either two binaries, a single star and a hierarchical triple system, two single stars and a binary, or four single stars. Among the dynamically ejected stars (or binaries), those with the lowest mass reach the highest velocities. In the first and third case, the surviving massive hard binary system(s) will normally only get a moderate velocity kick.

WR 20a could actually have formed in the centre of Westerlund 2 and might have ejected some lower mass stars. As a result of these interactions, WR 20a might have received a small recoil velocity that kicked it out of the cluster core. Considering an age of $1.5 \mathrm{Myr}$ for WR 20a (see Sect. 6.3) and a projected distance from the cluster core of $1.1 \mathrm{pc}$ (see above), a strict lower limit on the average velocity required for WR 20a to reach its present position would be $0.5-1.0 \mathrm{~km} \mathrm{~s}^{-1}$. Such a rather low value (of the same order as the rms velocity in a typical young open cluster) would most probably not be sufficient to make the binary leave the cluster. Nevertheless, the initial "ejection" velocity could have been significantly larger and a detailed model of the cluster's gravitational potential should allow to estimate the binary's present-day velocity. However, since we are lacking many ingredients (the total 
mass of the stars in the cluster, the total amount of gas in the cluster core, the exact 3D location of WR 20a with respect to Westerlund $2, \ldots$ ) building such a model is beyond the scope of the present paper. In any case, we note that systemic velocities of the order of (or even a factor of a few larger than) a typical cluster rms velocity are well below the detection threshold (especially for a WR binary for which it is almost impossible to establish the true systemic velocity). The less massive stars that got ejected through dynamical interactions with WR 20a would have travelled at significantly larger velocities (average velocities of $\geq 10-100 \mathrm{~km} \mathrm{~s}^{-1}$ ) and would probably be many parsecs away from the cluster centre by now.

Finally, it should be stressed that the radio emission of the RCW $49 \mathrm{H}$ II region reveals two wind-blown bubbles, one around WR 20b (WN7; Shara et al. 1991) and the other (blisterlike structure) around the Westerlund 2 cluster (Whiteoak \& Uchida 1997). Whiteoak \& Uchida suggested that the mechanical luminosity of the WR 20a wind exceeds the combined mechanical luminosity of the other stars in Westerlund 2 by a factor of about three. The dynamics of the latter bubble would therefore be largely dictated by WR 20a. However, Whiteoak \& Uchida (1997) estimated that the Lyman continuum flux provided by the known early-type stars in Westerlund 2 could only account for the observed radio flux of RCW 49, if the distance of RCW 49 were of order $2.3 \mathrm{kpc}$, i.e. much smaller than other estimates of the distance towards Westerlund 2 (see Sect. 6.2.2).

Whiteoak \& Uchida evaluated the ionising flux required to explain the entire radio flux density of RCW 49 (210 Jy at $0.843 \mathrm{GHz}$ ). If instead we consider only the shell around Westerlund 2, the radio flux density at $0.843 \mathrm{GHz}$ amounts to $110 \mathrm{Jy}$. Using the formalism of Martín-Hernández et al. (2003) and adopting an electron temperature of $7300 \mathrm{~K}$ (Whiteoak \& Uchida 1997) and $d=7.9 \mathrm{kpc}$, we infer a Lyman continuum photon flux of $\sim 3.0 \times 10^{50} \mathrm{~s}^{-1}$. Moffat et al. (1991) classified the spectra of two O6:V and five $07: \mathrm{V}$ stars in Westerlund 2 and from their photometry, we estimate that the cluster contains at least two more $07 \mathrm{~V}$ stars. According to the model grids of Smith et al. (2002), we find that these objects together provide an ionising photon flux of about $9.5 \times 10^{49} \mathrm{~s}^{-1}$. For the two WN6ha stars in WR 20a, we evaluate a total ionising flux of $1.45 \times 10^{50} \mathrm{~s}^{-1}$. Adding up these numbers, and taking into account the likely presence of a population of somewhat later O-type stars as well as a correction for an unknown fraction of binaries, we see that the early-type stars of Westerlund 2 could probably at least account for the ionisation of this shell. Once again, we emphasize the need for a detailed investigation of the stellar content of Westerlund 2 to address this issue.

\subsection{The evolutionary status of WR20a}

Since WR 20a is a close binary system with a rather short period (3.686 days), one could expect a priori that Roche lobe overflow (RLOF) might have played a role in its evolution. For the most massive stars $\left(M \geq 50 M_{\odot}\right)$ in close binaries however, it is likely that they evolve through a Luminous Blue Variable (LBV) phase (Vanbeveren 1991; Langer 1995) associated with

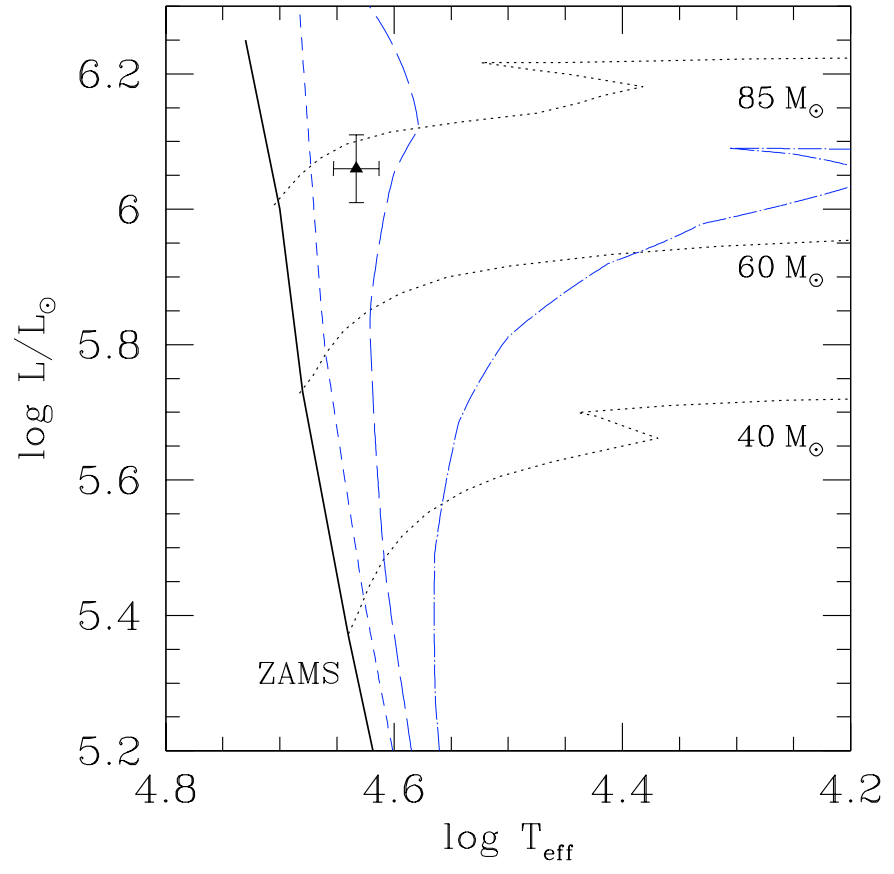

Fig. 9. Position of the components of WR 20a in the HertzsprungRussell diagram. The evolutionary tracks for single stars of initial mass 40,60 and $85 M_{\odot}$ as well as the isochrones corresponding to ages of 1,2 and $3 \mathrm{Myr}$ are from Schaller et al. (1992) for metallicity $Z=Z_{\odot}$.

a stellar wind mass-loss large enough to avoid mass transfer. As a result of this strong mass-loss, the binary period increases and at the end of the LBV phase, the binary should have a period longer than $\sim 10$ days. The LBV phase would correspond to the hydrogen shell burning phase and the post-LBV star should thus be a core helium burning WN star with an atmospheric hydrogen abundance of $X_{\text {atm }} \sim 0.2-0.3$ (Vanbeveren 1991).

In the context of this scenario, it seems more likely that the WN6ha stars in WR 20a are in a pre-LBV rather than postLBV evolutionary stage. In fact, no LBV ejected nebula around WR 20a has been reported in the literature, the current orbital period of the system is too short for a post-LBV system (see above) and the atmospheric hydrogen fraction is significantly larger than the value expected at the end of an LBV phase. Therefore, the WN6ha stars in WR 20a are likely to be core hydrogen burning stars and their evolution to date should have been similar to that of single stars of equal mass.

The location of the WN6ha components of WR 20a in the Hertzsprung-Russell diagram is illustrated in Fig. 9 along with the single star, solar metallicity and standard mass-loss rate, evolutionary models of Schaller et al. (1992). These evolutionary tracks account for the effect of mass-loss but do not include rotational mixing or magnetic fields.

Assuming that the components of WR 20a are currently evolving redwards in the HRD, we find that they lie between the 60 and $85 M_{\odot}$ initial mass evolutionary tracks. The stars appear to have evolved off the zero age main sequence and are actually between the 1 and $2 \mathrm{Myr}$ isochrones in the Schaller et al. models. The agreement between the age as determined from the single star isochrones and the age estimates for the 
Westerlund 2 cluster (Carraro \& Munari 2004; Piatti et al. 1998 ) is surprisingly good. A crude interpolation between the various models of Schaller et al. (1992) yields an evolutionary mass of $(76.6 \pm 4.0) M_{\odot}$, which is slightly lower than the observationally determined masses. A major difference concerns the chemical composition of the stars. Whilst the single star tracks of Schaller et al. do not predict any peculiar surface abundances for a star of this mass and age, the spectrum of WR 20a clearly reveals enhanced nitrogen and depleted carbon abundances. Could this be a result of rapid rotation $\left(v_{\text {eq }} \geq 300 \mathrm{~km} \mathrm{~s}^{-1}\right)$ leading to an efficient rotational mixing (Meynet \& Maeder 2000)? Assuming synchronous rotation in WR 20a, we estimate $v_{\text {eq }} \sim 265 \mathrm{~km} \mathrm{~s}^{-1}$ from the current orbital period and adopting a mean radius of $19.3 R_{\odot}$. Such a rotation velocity is probably sufficient to produce an efficient mixing that might contribute to the chemical enrichment of the stellar atmosphere.

Alternatively, the temperature and luminosity of the components of WR 20a could also be consistent with those of a single star evolving back towards the blue. However there are several reasons why this cannot be the case for WR 20a. In fact, in this case, the stars would fall almost exactly on the blue-wards loop of a star with initial mass $60 M_{\odot}$, but the current evolutionary mass of a star on this track would only be $\sim 30 M_{\odot}$, much less than observed, and the atmospheric hydrogen abundance predicted by this model would be $X_{\text {atm }} \leq 0.2$, significantly lower than what we find for WR 20a. Moreover, if the stars were currently evolving blue-wards, they should have reached large radii sometimes during their red-wards excursion and as a result they would have gone through a RLOF phase.

Finally, we note that if the initial orbital period is short enough, very massive stars in binary systems could evolve through a case-A RLOF (i.e. mass exchange would occur while the primary would still be in the core hydrogen burning phase). In the case of WR 20a, this is unlikely to be the case since the properties of the mass gainer and the mass loser should be quite different in a post case-A RLOF system, whereas the components of WR 20a have (almost) identical properties.

In summary, the current evolutionary status of the WN6ha stars in WR 20a would be most readily explained if the stars were core hydrogen burning objects in a pre-LBV phase (Vanbeveren 1991). The observed atmospheric chemical composition is likely a result of an efficient rotational mixing due to the large rotational velocities. While WR 20a has most probably not been affected by Roche lobe overflow, we emphasize that there is however one major difference between the evolution of the components of WR 20a and that of a single star of equal mass. In fact, whereas the rotational velocity of a very massive single star is expected to decrease rapidly as a result of the removal of angular momentum by the stellar wind (Meynet $\&$ Maeder 2000), the situation in a (synchronised) close binary is probably somewhat different since the evolution of the stellar rotation velocity is also coupled to that of the angular momentum of the orbital motion. Tassoul (1987; see also Tassoul \& Tassoul 1990) investigated the effect of tidally induced meridional circulation that tends to synchronise the rotational and orbital motions. Using Eq. (10) of Tassoul (1987), we estimate a spin-down (or spin-up) time scale of about $40 \mathrm{yrs}$ for the components of WR 20a. Even if the actual synchronisation time were an order of magnitude larger than this spin-down time, it would still be sufficiently short so that it seems plausible that both stars could achieve a high degree of synchronism. Therefore, we could expect that the rotational velocity might remain quite large over a longer time scale and the rotational mixing might thus be even more efficient than in the case of a single star.

\section{Summary and concluding remarks}

We have analysed the optical spectrum of the very massive binary WR 20a. We find that the $\mathrm{H} \alpha$ and He II $\lambda 4686$ emission lines have very similar profiles and display strong phase-locked variations suggesting that part of the line emission probably arises in a wind interaction region between the stars.

Combining photometric and spectroscopic measurements, we derive a reddening of $A_{\mathrm{V}} \simeq 6.0$ and a distance of $\sim 7.9 \mathrm{kpc}$. While the latter result is in reasonable agreement with the distance estimates of the Westerlund 2 cluster, it is significantly larger than most distance estimates for the H II region RCW 49. In order to solve this issue, a detailed census of the stellar population (spectral types and multiplicity) of Westerlund 2 is urgently needed. Such a study should not only help explain the ionisation of RCW 49, but should also provide clues on the formation of a very massive binary such as WR 20a and on its relation to Westerlund 2.

WR 20a is clearly detected in the X-rays, although the exact X-ray luminosity and its variability will only be determined by future X-ray observations, combining high sensitivity and a sufficient angular resolution to resolve WR 20a from the emission of Westerlund 2 and covering a large part of the orbital cycle.

We have used a model atmosphere code to derive some fundamental parameters of the WN6ha components in WR 20a. While we find that nitrogen is clearly enhanced and carbon is depleted in the atmospheres of these stars, little can be said about other elements with the present data set. More constraints on the chemical composition might come from future high-resolution high-quality optical spectra as well as UV observations.

Acknowledgements. It is a pleasure to thank Dr. D. Vanbeveren for discussion on massive close binary evolution, Dr. I. A. Bonnell for comments on dynamical interactions in open clusters, Dr. G. Carraro for providing us with his calibrated photometry of Westerlund 2 as well as Drs. L. K. Townsley and M. Tsujimoto for sharing their Chandra results with us prior to publication. We are also grateful to Dr. A. Moffat for discussion and to the referee Dr. K. Gayley for valuable comments that helped to improve our manuscript. The Liège team is greatly indebted to the Fonds National de la Recherche Scientifique (Belgium) for multiple assistance. This research is also supported in part by contract P5/36 "Pôle d'Attraction Interuniversitaire" (Belgian Federal Science Policy Office) and through the PRODEX XMM-OM and INTEGRAL projects. The SIMBAD database has been consulted for the bibliography. 


\section{References}

Berghöfer, T. W., Schmitt, J. H. M. M., Danner, R., \& Cassinelli, J. P. 1997, A\&A, 322, 167

Bonanos, A. Z., Stanek, K. Z., Udalski, A., et al. 2004, ApJ, 611, L33

Bonnell, I. A. 2004, private communication

Bonnell, I. A., \& Bate, M. R. 2002, MNRAS, 336, 659

Bonnell, I. A., Bate, M. R., \& Zinnecker, H. 1998, MNRAS, 298, 93

Bonnell, I. A., Vine, S. G., \& Bate, M. R. 2004, MNRAS, 349, 735

Carraro, G. 2004, private communication

Carraro, G., \& Munari, U. 2004, MNRAS, 347, 625

Churchwell, E., Whitney, B. A., Babler, B. L., et al. 2004, ApJS, 154, 322

Clark, J. S., et al. 2004, in preparation

Crowther, P. A., Hillier, D. J., \& Smith, L. J. 1995, A\&A, 293, 403

Crowther, P. A., \& Dessart, L. 1998, MNRAS, 296, 622

Gayley, K. G., Owocki, S. P., \& Cranmer, S. R. 1997, ApJ, 475, 786

Gayley, K. G., Owocki, S. P., \& Cranmer, S. R. 1999, ApJ, 513, 442

Goldwurm, A., Caraveo, P. A., \& Bignami, G. F. 1987, ApJ, 322, 349

Gosset, E., Nazé, Y., Claeskens, J.-F., et al. 2005, A\&A, 429, 685

Hamuy, M., Walker, A. R., Suntzeff, N. B., et al. 1992, PASP, 104, 533

Herbig, G. 1995, ARA\&A, 33, 19

Hillier, D. J., \& Miller, D. L. 1998, ApJ, 496, 407

Horne, K. 1991, in Fundamental Properties of Cataclysmic Variable Stars: 12th North American Workshop on Cataclysmic Variables and Low Mass X-ray Binaries (SDSU Press), ed. A. W. Shafter, 23

Langer, N. 1995, in Wolf-Rayet Stars: Binaries, Colliding Winds, Evolution, ed. K. A. van der Hucht, \& P. M. Williams, Proc. IAU Symp., 163, 15

Leonard, P. J. T., \& Duncan, M. J. 1990, AJ, 99, 608

Lubow, S. H., \& Shu, F. H. 1975, ApJ, 198, 383

Martín-Hernández, N. L., van der Hulst, J. M., \& Tielens, A. G. G. M. 2003, A\&A, 407, 957

Mereghetti, S., \& Belloni, T. 1995, in Wolf-Rayet Stars: Binaries, Colliding Winds, Evolution, ed. K. A. van der Hucht, \& P. M. Williams, Proc. IAU Symp., 163, 76

Mereghetti, S., \& Belloni, T. 1996, in Röntgenstrahlung from the Universe, ed. H. U. Zimmermann, J. Trümper, \& H. Yorke, MPE Rep., 263, 53

Meynet, G., \& Maeder, A. 2000, A\&A, 361, 101

Moffat, A. F. J., Shara, M. M., \& Potter, M. 1991, AJ, 102, 642

Moffat, A. F. J., Corcoran, M. F., Stevens, I. R., et al. 2002, ApJ, 573, 191

Morris, P. W., Brownsberger, K. R., Conti, P. S., Massey, P., \& Vacca, W. D. 1993, ApJ, 412, 324

Piatti, A. E., Bica, E., \& Clariá, J. J. 1998, A\&AS, 127, 423
Pittard, J. M., \& Stevens, I. R. 2002, A\&A, 388, L20

Pollock, A. M. T., Haberl, F., \& Corcoran, M. F. 1995, in WolfRayet Stars: Binaries, Colliding Winds, Evolution, ed. K. A. van der Hucht, \& P. M. Williams, Proc. IAU Symp., 163, 512

Portegies Zwart, S. F., Makino, J., McMillan, S. L. W., \& Hut, P. 1999, A\&A, 348, 117

Raassen, A. J. J., van der Hucht, K. A., Mewe, R., et al. 2003, A\&A, 402,653

Raboud, D., \& Mermilliod, J.-C. 1998, A\&A, 333, 897

Rauw, G., Nazé, Y., Carrier, F., et al. 2001, A\&A, 368, 212

Rauw, G., Crowther, P. A., Eenens, P. R. J., Manfroid, J., \& Vreux, J.-M. 2002, A\&A, 392, 563

Rauw, G., De Becker, M., Nazé, Y., et al. 2004, A\&A, 420, L9

Sagar, R., Munari, U., \& de Boer, K. S. 2001, MNRAS, 327, 23

Savage, B. D., \& Mathis, J. S. 1979, ARA\&A, 17, 73

Schaller, G., Schaerer, D., Meynet, G., \& Maeder, A. 1992, A\&AS, 96, 269

Shara, M. M., Moffat, A. F. J., Smith, L. F., \& Potter, M. 1991, AJ, 102,716

Shara, M. M., Moffat, A. F. J., Smith, L. F., et al. 1999, AJ, 118, 390

Skrutskie, M. F., Schneider, S. E., Stiening, R., et al. 1997, in The Impact of Large Scale Near-IR Sky Surveys, ed. F. Garzon et al. (Dordrecht: Kluwer Acad. Pub.), 25

Smith, L. F. 1968, MNRAS, 140, 409

Smith, L. J., Norris, R. P. F., \& Crowther, P. A. 2002, MNRAS, 337, 1309

Stevens, I. R. 1988, MNRAS, 235, 523

Stevens, I. R., \& Pollock, A. M. T. 1994, MNRAS, 269, 226

Stevens, I. R., Blondin, J. M., \& Pollock, A. M. T. 1992, ApJ, 386, 265

Stone, R. P. S., \& Baldwin, J. A. 1983, MNRAS, 204, 347

Tassoul, J.-L. 1987, ApJ, 322, 856

Tassoul, J.-L., \& Tassoul, M. 1990, ApJ, 359, 155

Thaller, M. L., Gies, D. R., Fullerton, A. W., Kaper, L., \& Wiemker, R. 2001, ApJ, 554, 1070

Townsley, L. K., Feigelson, E. D., Montmerle, T., et al. 2004, in X-ray and Radio Connections [arXiv: astro-ph/0406349]

Tsujimoto, M., Townsley, L. K., Feigelson, E. D., et al. 2004, ApJ, submitted

Vanbeveren, D. 1991, A\&A, 252, 159

van der Hucht, K. A. 2001, New Astron. Rev., 45, 135

Walborn, N. R., Morrell, N. I., Howarth, I. D., et al. 2004, ApJ, 608, 1028

Wessolowski, U. 1996, in Röntgenstrahlung from the Universe, ed. H. U. Zimmermann, J. Trümper, \& H. Yorke, MPE Rep., 263, 75 Whiteoak, J. B. Z., \& Uchida, K. I. 1997, A\&A, 317, 563 